ARTICLE

DOI: $10.1038 /$ s41467-018-07563-6

\title{
Widespread bacterial lysine degradation proceeding via glutarate and L-2-hydroxyglutarate
}

Sebastian Knorr ${ }^{1,2}$, Malte Sinn¹,2, Dmitry Galetskiy ${ }^{1}$, Rhys M. Williams ${ }^{3}$, Changhao Wang ${ }^{1}$ Nicolai Müller ${ }^{3}$, Olga Mayans ${ }^{2,3}$, David Schleheck ${ }^{2,3} \&$ Jörg S. Hartig ${ }^{1,2}$

Lysine degradation has remained elusive in many organisms including Escherichia coli. Here we report catabolism of lysine to succinate in E. coli involving glutarate and L-2-hydroxyglutarate as intermediates. We show that CsiD acts as an $\alpha$-ketoglutarate-dependent dioxygenase catalysing hydroxylation of glutarate to L-2-hydroxyglutarate. CsiD is found widespread in bacteria. We present crystal structures of CsiD in complex with glutarate, succinate, and the inhibitor $\mathrm{N}$-oxalyl-glycine, demonstrating strong discrimination between the structurally related ligands. We show that $L$-2-hydroxyglutarate is converted to $\alpha$ ketoglutarate by LhgO acting as a membrane-bound, ubiquinone-linked dehydrogenase. Lysine enters the pathway via 5-aminovalerate by the promiscuous enzymes GabT and GabD. We demonstrate that repression of the pathway by CsiR is relieved upon glutarate binding. In conclusion, lysine degradation provides an important link in central metabolism. Our results imply the gut microbiome as a potential source of glutarate and L-2-hydroxyglutarate associated with human diseases such as cancer and organic acidurias.

\footnotetext{
${ }^{1}$ Department of Chemistry, University of Konstanz, Konstanz 78457, Germany. ${ }^{2}$ Konstanz Research School Chemical Biology (KoRS-CB), Konstanz 78457 , Germany. ${ }^{3}$ Department of Biology, University of Konstanz, Konstanz 78457, Germany. These authors contributed equally: Sebastian Knorr, Malte Sinn. Correspondence and requests for materials should be addressed to J.S.H. (email: joerg.hartig@uni-konstanz.de)
} 
F or many organisms, lysine degradation has remained as a white spot on the metabolic map. In E. coli, a lysine decarboxylase activity has been described ${ }^{1,2}$. Further, degradation of cadaverine (Cad) to glutarate (GA) has been proposed in pseudomonads in $1977^{3}$. We initiated our study when being interested in the function of Escherichia coli protein CsiD (carbon starvation induced protein D). Its gene $c s i D$ is the first of a fivegene operon in E. coli (csiD-lhgO-gabDTP) (illustrated in Fig. 1), whose expression and regulation has been studied in great detail. The whole operon is specifically induced in stationary phase (carbon starvation) while the gabDTP genes are induced also separately in response to nitrogen starvation. Immediately downstream of the operon, $c s i R$ encodes a transcription factor that represses the $c s i D$ operon ${ }^{4}$. In addition to CsiR, the $c s i D$ operon is controlled by cAMP-CRP, leu-LRP and H-NS ${ }^{5,6}$. The $\mathrm{CsiD}$ protein belongs to the non-haem $\mathrm{Fe}(\mathrm{II})$-dependent oxygenase family (protein family PF08943), but the native substrate(s) of the predicted enzyme and its role during stationary phase of $E$. coli remained unknown. A crystal structure of CsiD that was solved in a structural genomics effort suggested that CsiD functions as an $\alpha$-ketoglutarate ( $\alpha \mathrm{KG})$-dependent dioxygenase ${ }^{7}$. Since the subsequent gene of the operon (lhgO, Fig. 1) has been described as an $L$-2-hydroxyglutarate (L2HG) oxidase ${ }^{8}$, we hypothesized whether CsiD may produce L2HG by hydroxylation of GA, a compound that has so far been considered as a 'deadend' metabolite ${ }^{9}$. Here we demonstrate that lysine is degraded via cadaverine to GA by a series of promiscuous aminotransferase and dehydrogenase reactions. GA is subsequently hydroxylated by $\mathrm{CsiD}$ and the product $\mathrm{L} 2 \mathrm{HG}$ is oxidised to $\mathrm{aKG}$ by the dehydrogenase LhgO that couples to the respiratory chain by reducing the quinone pool. Furthermore, we show that repression of the CsiD operon by the transcription factor CsiR is selectively relieved by glutarate.

\section{Results}

Characterisation of glutarate hydroxylase CsiD. We purified CsiD and demonstrated by NMR (Fig. 2a and Supplementary Figures 1,2) and LC-MS that it indeed hydroxylates GA to 2hydroxyglutarate, while the co-substrate $a \mathrm{KG}$ is converted to succinate (SA) (and $\mathrm{CO}_{2}$ ), as is common for this enzyme class $^{10,11}$. By derivatisation of the product with diacetyl-L-tartaric anhydride $^{12}$ we demonstrated that L2HG is produced in a highly stereospecific manner; no $D$-2-hydroxyglutarate was detectable (Supplementary Figure 3). This finding is in accordance with the reported specificity for the $L$-enantiomer of the subsequent LhgOcatalysed reaction ${ }^{8}$. When the reaction was measured with a Clark $\mathrm{O}_{2}$ electrode, we determined a specific activity of $53+/-3$ $\mu \mathrm{mol} \mathrm{min} \mathrm{mg}^{-1} \mathrm{mg}^{-1}$ and an apparent $K_{\mathrm{m}}=650+/-20 \mu \mathrm{M}$ for GA and $K_{\mathrm{m}}=100+/-7 \mu \mathrm{M}$ for $\alpha \mathrm{KG}$ (Fig. $2 \mathrm{~b}, \mathrm{c}$ ). Other dicarboxylic acids (oxalate, malonate, SA, adipate, and pimelate tested) were not converted. The physiological role of $\mathrm{CsiD}$ as glutaratemetabolising enzyme in the stationary phase of E. coli was confirmed when we tested its $c s i D$ knockout strain $(\Delta c s i D)$ by LC-MS of small-molecule extracts: with carbon starvation and entry into the stationary phase, the intracellular concentration of GA accumulated to much higher levels in the $\Delta c s i D$ strain than compared to the wildtype (Fig. 2d). While it is commonly encoded in Enterobacteriaceae, CsiD is found also in genomes of many other proteobacteria and bacilli (Supplementary Figure 4).

$\mathrm{CsiD}$ as characterised glutarate hydroxylase is also interesting with respect to the two structurally similar substrates GA and aKG. In addition, the product of the reaction, L2HG, is known as an oncometabolite inhibiting aKG-dependent dioxygenases such as TET-type and Jmjc-type demethylases ${ }^{13,14}$. We did observe weak inhibition of CsiD by its product L2HG (Supplementary
Figure 5) in contrast to the aforementioned representatives of the same enzyme class. In order to shed more light on this interesting finding, we solved the atomic structure of CsiD in complex with its substrate (GA), its product (SA) as well as in complex with the aKG-analog N-oxalylglycine (NOG) as inhibitor, by X-ray crystallography (Fig. 2e, Extended Fig. 6, Supplementary Table 1). The crystal form obtained (with symmetry P42 ${ }_{1} 2$ ) contains two molecular copies of CsiD in its asymmetric unit. These two noncrystallographic copies are identical (RMSD $=0.082 \AA)$. The biological tetrameric form of $\mathrm{CsiD}$ is generated by the symmetry of the crystallographic lattice, as it was previously the case in structures lacking the by then unknown substrate 7,15 . The enzyme protomer adopts a distorted jelly-roll fold composed of a $\beta$-sheet core flanked by $\alpha$-helices, as previously described. The iron ion is bound to the active site of CsiD by residues His160, Asp162, and His292 and three solvent molecules that complete an octahedral coordination sphere (Supplementary Figure 6a). In ligand-bound structures, one or more solvent molecules become replaced by interacting oxygen atoms from the ligands.

Ligand-bound CsiD structures revealed two binding sites located at opposite sides of the $\mathrm{Fe}^{2+}$ ion (Fig. $2 \mathrm{e}$ and Supplementary Figure $6 \mathrm{~b}-\mathrm{d}$ ). In the substrate site (here termed site I), GA is directly coordinated by the $\mathrm{Fe}^{2+}$ ion via an oxygen atom from one of its terminal carboxyl groups. The other carboxyl group forms a salt bridge with a conserved residue Arg311 and the main chain nitrogen of Gly163 (Supplementary Figure 6b). The co-substrate site (here termed site II) is seen in crystal structures in this work occupied by (a) the nonprocessable aKG-analogue NOG (where atom C3 is substituted by nitrogen compared to $\alpha \mathrm{KG}$ ) or (b) the co-product SA. NOG binds the $\mathrm{Fe}^{2+}$ ion via the oxo- and a terminal oxygen atom of its oxalyl moiety as well as forming a salt bridge with residue Arg309 (Supplementary Figure 6c). SA is bound to $\mathrm{Fe}^{2+}$ via a terminal carboxyl group in a bidentate fashion (Supplementary Figure 6d) and further interacts with Arg309 through a low-occupancy solvent molecule that occupies the site that is generated by decarboxylation of $a \mathrm{KG}$ to $\mathrm{SA}$, mimicking the interaction to the NOG oxygen in that complex. Conformational changes in the CsiD protein were not observed in any of the complexed structures, independently of whether ligand site I or site II was occupied. Importantly, in structures with site I or II occupied, always the cognate ligand for the respective site was observed with the other site unoccupied. This finding demonstrates a high degree of specificity of CsiD regarding the binding of the structurally similar substrate, co-substrate, and products.

LhgO is a membrane bound quinone-dependent oxidoreductase. We next considered the fate of L2HG in E. coli. The second protein of the operon, LhgO, has been described as a FAD-dependent $L$-2-hydroxyglutarate oxidase, which utilizes molecular oxygen to yield $\alpha \mathrm{KG}$ and hydrogen peroxide ${ }^{8}$. However, we could not detect $\mathrm{O}_{2}$ consumption with L2HG using the purified recombinant and active (see below) LhgO enzyme. LhgO is found very widespread with homologs in most eubacteria, archaea, and eukaryotes. The human (41\% identity with $E$. coli LhgO over full amino acid sequence) and A. thaliana homologs are described as $\mathrm{L} 2 \mathrm{HG}$ dehydrogenases ${ }^{16,17}$ that localize to the inner mitochondrial membrane. Further, for the plant enzyme a coupling of the electron transfer to the respiratory chain has been proposed $^{17}$.

Hence, we tested whether the $E$. coli LhgO protein may be a dehydrogenase that feeds the high-energy electrons (reported $\mathrm{E}^{0}$ $=19+/-8 \mathrm{mV})^{8}$ from the oxidation of L2HG via ubiquinone to the respiratory electron transport chain (Fig. 1): Purified recombinant LhgO directly reduced ubiquinone (Supplementary 


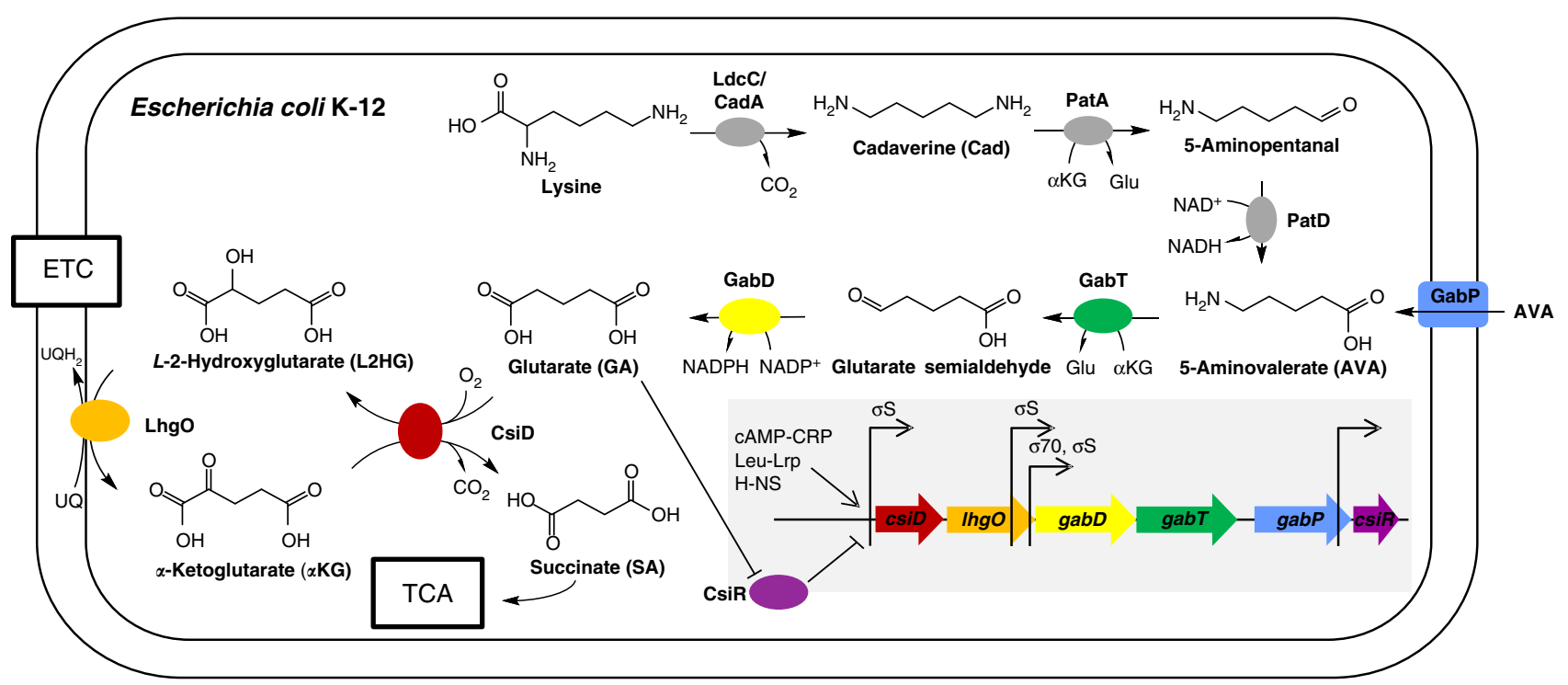

Fig. 1 Catabolism of lysine to succinate in Escherichia coli K-12. Summary of the central metabolic pathway discovered in this study and of the corresponding gene cluster with its regulation (grey inset) in E. coli. We show that E. coli CsiD (carbon starvation induced protein D) functions as a $\alpha$-ketoglutaratedependent, $\mathrm{CO}_{2}$ - and succinate-forming glutarate hydroxylase, which produces L-2-hydroxyglutarate, and that E. coli LhgO ( $L$-2-hydroxyglutarate oxidase) acts as a membrane-bound, ubiquinone-linked, $\alpha$-ketoglutarate-producing $L$-2-hydroxyglutarate dehydrogenase. Effectively, glutarate is converted to succinate by these two reactions. We also show that $E$. coli feeds glutarate into these reactions from 5 -aminovalerate by promiscuous $\mathrm{GabT}$, when acting as 5-aminovalerate transaminase, and by promiscuous GabD, when acting as glutarate seminaldehyde dehydrogenase. Thus, these GabT-GabD-CsiD-LhgO reactions link the central metabolism (TCA cycle) with the yet incompletely known lysine catabolism in E. coli, that is, with LdcC and CadA as lysine decarboxylases, PatA (putrescine transaminase) acting as cadaverine transaminase and PatD as 5-aminopentanal dehydrogenase $1,2,26,28$. The catabolism of lysine by this pathway is specifically induced in the stationary phase of $E$. coli (see text). The inbox shows the structure and regulation of the csiD-lhgOgabD-gabT operon, which include also genes gabP encoding for a gamma-aminobutyrate (GABA) transporter that also imports the C5-homologue 5aminovalerate 55 and csiR encoding for a ligand-dependent transcription factor. Arrows represent transcription start sites (TSS) of different transcripts. Transcription of the operon is known to be enhanced by regulators CAMP-CRP, leu-LRP and H-NS5, 6 , and repressed by CsiR ${ }^{4}$. We demonstrated that this repression is relieved specifically upon binding of glutarate to CsiR

Figure 7) in a L2HG-dependent manner with a specific activity of $0.33+/-0.002 \mu \mathrm{mol} \mathrm{min}^{-1} \mathrm{mg}^{-1}$. Enzyme activity was not enhanced by supplementing FAD to the reaction. Together with the yellow colour of purified LhgO this indicates that bound FAD was co-purified saturating the reaction. Further, this activity of native LhgO in E. coli was found exclusively in the membrane fraction but not in the soluble protein fraction (Fig. 3a), and a $\Delta$ lhgO strain completely lacked the activity. Additionally, the redox activity of membranes upon L2HG addition was restored in a lhgO+ complementation strain, but the activity was lower compared to WT E. coli membranes; this may be explained by inefficient incorporation of recombinant LhgO into the membrane, possibly caused by the His-Tag. Furthermore, we were able to show that the natural quinones of $E$. coli can serve as electron acceptors for LhgO. Membrane fractions of E. coli WT were incubated with L2HG or SA (testing succinate dehydrogenase as positive control) as substrates for the respective dehydrogenases. The ratio between ubiquinol to ubiquinone increased in the presence of both SA and L2HG compared to the control reaction without added substrate, indicating that electron transfer from these substrates to ubiquinone occurred in the membrane fractions (Fig. 3b). The ubiquinol/ubiquinone pool was unaffected by L2HG in a membrane fraction prepared from an $E$. coli strain lacking $\mathrm{LhgO}(\Delta l h g O)$. We exclusively detected menaquinone and demethylmenaquinone in their oxidised but not reduced forms. However, many ETC-coupled dehydrogenases electron chain in E. coli show a wider substrate range for various quinone species and also under aerobic conditions, naphtoquinones can be used as electron acceptors ${ }^{18,19}$. Notably, since menaquinole and demethylmenaquinole reoxidise quickly ${ }^{20}$ and might not be detectable in our assay, we cannot exclude that LhgO may accept also these quinones as electron acceptors. However, when 2,6chloroindophenol (DCPIP) was used as artificial electron acceptor both native LhgO in the membrane fraction and recombinant LhgO revealed higher activities in the presence of ubiquinone $_{1}$ compared to menaquinone ${ }_{4}$ (Fig. 3c). Addition of the respiratory chain inhibitor 2-heptyl-4-hydroxyquinoline $n$ oxide (HQNO) in concentrations higher than $80 \mu \mathrm{M}$ to membrane fractions abolished $\mathrm{O}_{2}$ consumption and DCPIP activity, again confirming coupling of LhgO to the respiratory chain (Fig. 3d). Taking into account that for E. coli ubiquinone is the main electron acceptor under aerobic conditions ${ }^{19}$, these results support the conclusion that LhgO acts as a quinonedependent oxidoreductase located in the cytoplasmic membrane.

The degradation of lysine to glutarate. We next considered the source of GA, a compound that has not yet been shown to play an important and widespread role in metabolism. In E. coli, csiD and lhgO are co-encoded with genes for $\gamma$-aminobutyrate (GABA) shunt enzymes ( $g a b T$ and gabD, Fig. 1). GabT is described as GABA transaminase that yields succinic semialdehyde. GabD is a dehydrogenase that converts succinic semialdehyde to $\mathrm{SA}^{21}$. We hypothesized whether GabT may convert also the C5-homolog 5aminovalerate (AVA) to glutarate semialdehyde and whether GabD converts the glutarate semialdehyde to GA (Fig. 1), as it has been described for Pseudomonas 22 and C. glutamicum $^{23,24}$ homologues. We purified GabT and GabD from E. coli and demonstrated that they use AVA with comparable efficiency to GABA $\left(K_{\mathrm{M}}^{\mathrm{GABA}}=197 \pm 27 \mu \mathrm{M}, K_{\mathrm{M}}^{\mathrm{AVA}}=439 \pm 29 \mu \mathrm{M}\right)$ for a coupled GabT/D reaction, (Fig. 4a). The reaction was additionally analysed via high-resolution mass spectrometry to confirm GA as the product derived from AVA (Supplementary Figure 8). 
a

aKG GA<smiles>O=C(O)CCCCCC(=O)O</smiles>
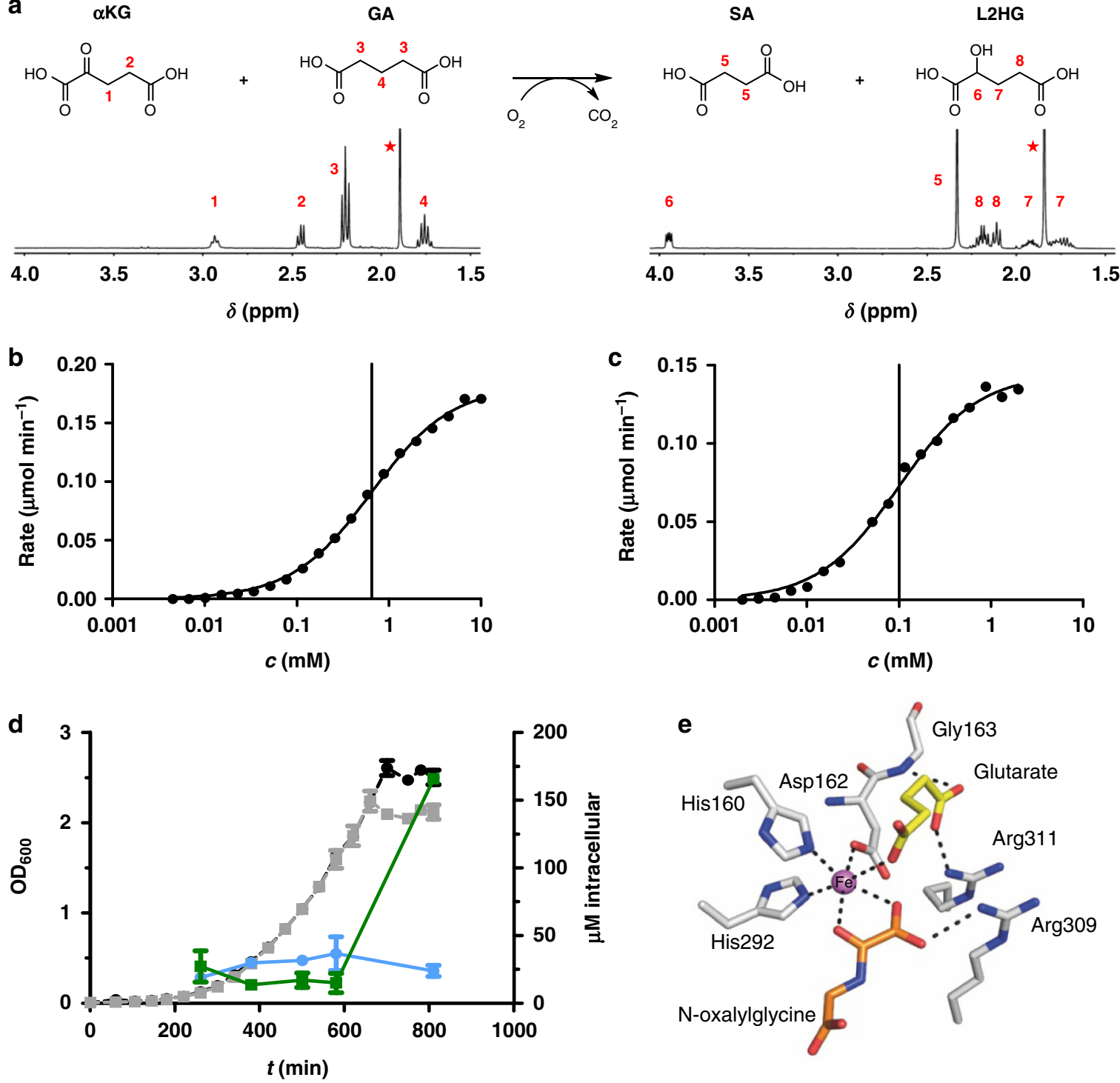

Fig. 2 CsiD is an $\alpha K G$-dependent dioxygenase converting GA to L2HG. a Reaction as catalysed by CsiD and its characterisation by NMR. CsiD converts GA and $\alpha K G$ to $L 2 H G$ and SA, respectively (and carbon dioxide). ${ }^{1} \mathrm{H}-\mathrm{NMR}$ spectra of educts (left) and products (right) after overnight incubation with CsiD in ammonium acetate buffer are shown, asterisks: signals from acetate buffer. b, c Reaction rate, measured as consumption of $\mathrm{O}_{2}$ in a Clark-type oxygen electrode, is plotted against the concentration of GA (b) and $\alpha \mathrm{KG}(\mathbf{c})$. Data were fitted with Michaelis-Menten equation (solid line). $K_{\mathrm{M}}$ is indicated by the vertical line. $\mathbf{d}$ Intracellular GA concentrations in different growth phases of $E$. coli M9 cultures. Circles (WT E. coli strain) and squares ( $\Delta$ csiD E. coli strain) represent intracellular GA concentrations (blue: WT, green: $\Delta c s i D$, right $y$-axis). Bacterial cell growth is indicated by optical density at $600 \mathrm{~nm}\left(\mathrm{OD}_{600}\right)$ in black and grey (WT and $\Delta c s i D$, left $y$-axis). Shown are means of biological duplicates with error bars representing standard deviation including technical duplicates. e Crystal structure of CsiD. The active site of a protomer with substrate GA (yellow) and the $\alpha$ KG co-substrate analog N-oxalylglycine (orange) is shown. The model is a reconstruction obtained by superimposing individual crystal structures of CsiD bound to each of the ligands (the CsiD enzyme is equivalent in both structures; RMSD $=0.16 \AA$ ). The Fe ion is shown in magenta. Dashed lines represent interactions between ligands and residues in the binding pocket

For a potential source of AVA, we considered a pathway starting from lysine (Fig. 1), through decarboxylation of lysine to Cad followed by transaminase and dehydrogenase reactions. These reactions have been described in Pseudomonas species ${ }^{3}$. In E. coli, two lysine decarboxylases are known (CadA and $\mathrm{LdcC})^{1,2}$. Reactions starting from the $\mathrm{C} 4$ diamine putrescine are catalysed by PatA and PatD, resulting in GABA ${ }^{25-27}$. It has already been shown that the E. coli transaminase PatA can process Cad with the same activity as putrescine ${ }^{26}$. Since there is no data available concerning PatD producing AVA in E. coli, we compared coupled PatA/D activities for putrescine and Cad as described for GabT/D before. The coupled PatA/D reaction with Cad showed a $K_{\mathrm{M}}{ }^{\mathrm{Cad}}$ of $0.37 \pm 0.05 \mathrm{mM}$ and a $V_{\max }$ of $11.8 \mu \mathrm{M} \mathrm{min}^{-1}$, whereas PatA/D reaction with putrescine revealed a higher $K_{\mathrm{M}}{ }^{\text {Put }}$ of $1.43 \pm 0.07$ $\mathrm{mM}$ and a higher $V_{\max }$ of $29.1 \mu \mathrm{M} \mathrm{min}^{-1}$ (Fig. 4b). Furthermore, we confirmed the production of 5-aminopentanal (APA) and piperideine formed by PatA, as well as AVA by PatA/D via mass spectrometry analysis (Supplementary Figure 9). Our findings are further substantiated by the observation that overexpression of $l d c C$, pat $A$, and patD from E. coli can be used for the production of AVA from lysine in C. glutamicum ${ }^{28}$. An alternative sequence of reactions that could produce AVA from lysine degradation is the putrescine utilization pathway (puuABCD) of E. coli, which is proposed to degrade extracellular putrescine ${ }^{29}$. 

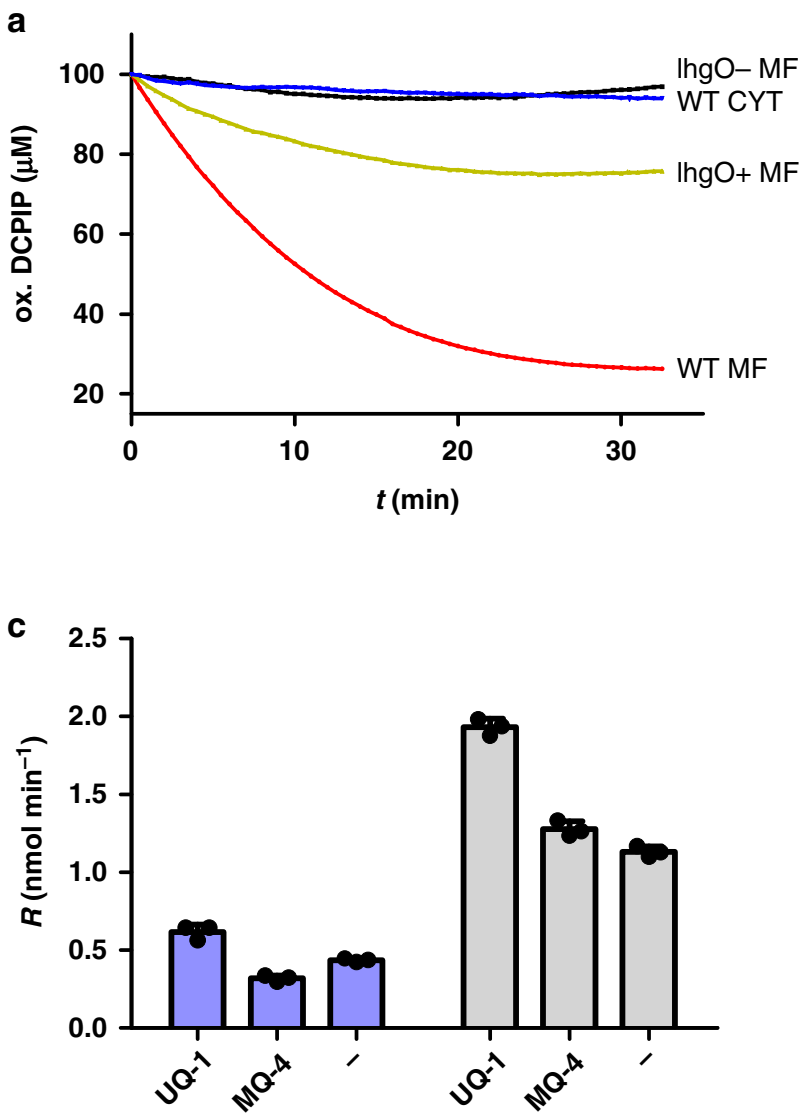

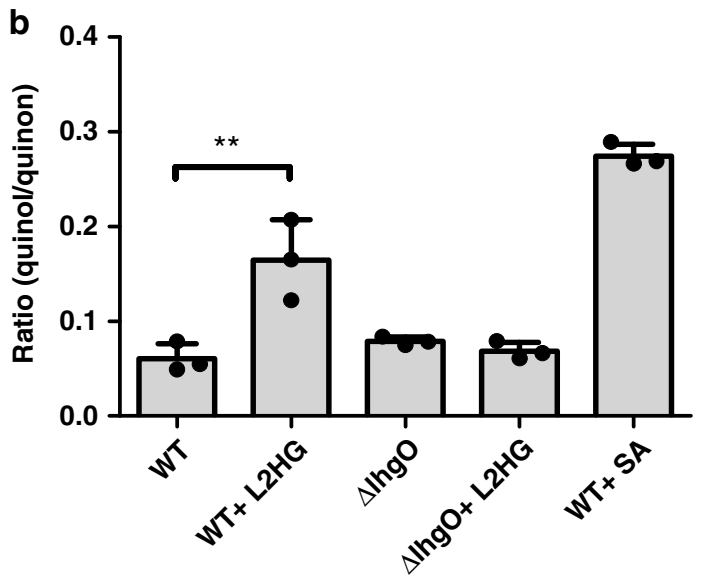

d

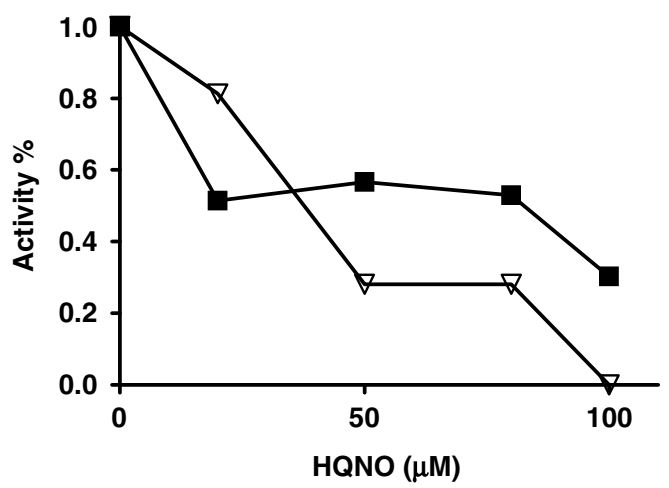

Fig. 3 LhgO as a membrane-associated, L2HG:quinone oxidoreductase in E. coli. a Activity of E. coli WT membrane fraction (red; WT MF) transfering electrons from L2HG to DCPIP compared to a WT cytosolic fraction (blue; WT CYT), a membrane fraction isolated from a $\triangle$ lhgO knockout strain (black; IhgO- MF) and a complementation strain of $\Delta / \mathrm{hgO}$ with recombinant LhgO (green, IhgO+MF). Depletion of the oxidized form of DCPIP over time was measured photometrically. $\mathbf{b}$ Ubiquinol to ubiquinone ratio (red./ox.) was measured in $E$. coli membrane fractions in the presence or absence of $\mathrm{L} 2 \mathrm{HG}$ or SA. Quinones were extracted from membrane fractions from either E. coli WT or $\Delta$ lhgO after the incubation with either L2HG or SA and measured by HPLC. Error bars represent standard deviations of triplicate experiments. Significance was assessed by a unpaired one-tailed Student's $t$-test ( ${ }^{\star \star} P$ value $=$ 0.0083). c Reaction rate $(R)$ of purified LhgO (blue columns) and E. coli WT membrane fraction (grey columns) measured by DCPIP reduction per time in dependence of ubiquinone $1\left(\mathrm{UQ}_{1}\right)$ or menaquinone-4 $\left(\mathrm{MQ}_{4}\right)$ after addition of $\mathrm{L} 2 \mathrm{HG},(-)$ no quinone added. Error bars represent standard deviations of triplicate experiments. $\mathbf{d}$ Inhibition of E. coli WT membrane activity by HQNO titration. HQNO was added before L2HG and rates were normalised to the activity without added HQNO. DCPIP reduction (spectrophotometrically) is indicated with black squares and oxygen consumption of membranes (Clark electrode) by white triangles

Validation of the complete degradation pathway. In order to validate the pathway in Fig. 1 we traced fully ${ }^{13} \mathrm{C}$-labelled and ${ }^{15} \mathrm{~N}$-labelled lysine in growth experiments. E. coli was grown in minimal medium with glucose as carbon (and energy) source and supplemented with isotope-labelled lysine, since lysine cannot be utilized as the sole carbon source by E. coli (for growth experiments utilizing the intermediates of the lysine degradation pathway as $\mathrm{C}$-sources and $\mathrm{N}$-sources see Supplementary Figure 10). We found high intracellular concentrations of Cad, APA, and GA predominantly in the fully labelled form (Fig. 5a). Although GA was detected only at $180 \mu \mathrm{M}$ in WT E. coli, GA levels accumulated to almost $5 \mathrm{mM}$ in a $\Delta c s i D$ strain. Importantly, Cad, APA, and GA were found predominantly in their completely labelled form. Fully ${ }^{13} \mathrm{C}$-labeled AVA was detectable at $640 \mu \mathrm{M}$ in the $\triangle g a b T$ strain. $\alpha \mathrm{KG}$ and SA were detected predominantly in non-labeled form in accordance with multiple sources of these central metabolites. In the $\Delta c s i D$ strain we found highly elevated levels of ${ }^{13} \mathrm{C}$-labeled GA but no ${ }^{13} \mathrm{C}$-labeled metabolites downstream of the CsiD reaction ( $\mathrm{SA}, \alpha \mathrm{KG})$. In accordance with SA as end product of the lysine degradation pathway, $[\mathrm{M}+4]$ and $[\mathrm{M}$ +2 -labelled SA was observed in small amounts. The labelling pattern of the detected intermediates support an unbranched pathway as depicted in Fig. 1. A detailed list of the intracellular concentrations and labelling patterns of all intermediates of the pathway can be found in extended data Table 3. Of note, we could also detect ${ }^{13} \mathrm{C}$-labeled GA in a $\triangle g a b T$ strain indicating that other transaminases besides GabT are able to process AVA. In E. coli, there are isoenzymes for both GabT and GabD known, namely the GABA transaminase PuuE and the succinate semialdehyde dehydrogenase Sad. We demonstrate for a coupled PuuE/Sad reaction with purified enzymes that similar to the findings with GabT/D (Fig. 4a) AVA can be used as a substrate (Supplementary Figure 11). Taken together, the isotope labelling experiments prove that lysine degradation as depicted in Fig. 1 is the source of GA in E. coli. It can be summed up as:

$$
\begin{gathered}
\text { L-lysine }+2 \alpha \mathrm{KG}+2 \mathrm{NAD}(\mathrm{P})^{+}+\mathrm{O}_{2}+\mathrm{UQ} \longrightarrow \\
\mathrm{SA}+2 \mathrm{CO}_{2}+2 \text { glutamate }+2 \mathrm{NAD}(\mathrm{P}) \mathrm{H}+\mathrm{UQH}_{2}
\end{gathered}
$$

Characterisation of the allosteric repressor CsiR. In E. coli, the $c s i D$ operon is followed by a transcription factor termed CsiR (Fig. 1) that has been shown previously to negatively regulate $c s i D$ operon expression ${ }^{4}$. Upon deletion of $c s i R$, the expression of the 
a
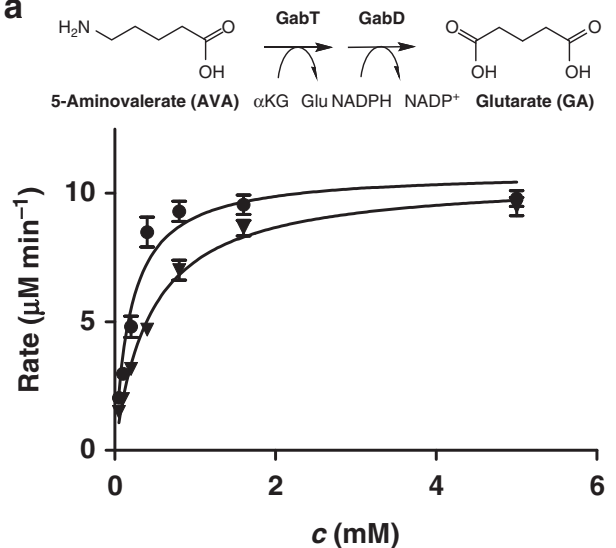

b

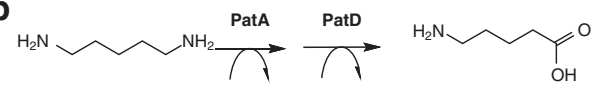

Cadaverine (Cad) $\alpha K G$ Glu $\mathrm{NAD}^{+} \mathrm{NADH}$ 5-Aminovalerate (AVA)

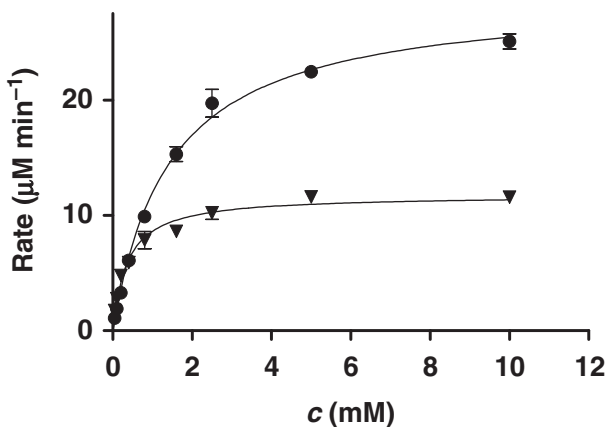

Fig. 4 Promiscuous transaminase/dehydrogenase pairs involved in lysine degradation. a Coupled GabT (GABA transaminase) and GabD (succinic

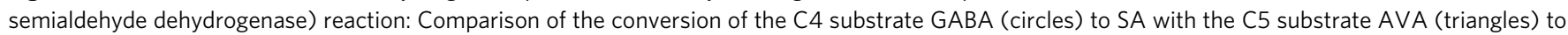
GA. b Coupled PatA (putrescine transaminase) and PatD ( $\gamma$-aminobutyraldehyd dehydrogenase) reaction: Comparison of the conversion of the C4 substrate putrescine (circles) to GABA with the C5 substrate Cad (triangles) to AVA. Reaction rate ( $\mu$ M/min) is shown as a function of substrate concentration. Rate is given as $\mu \mathrm{M} \mathrm{NADP}^{+} / \mathrm{NAD}^{+}$reduced per min, monitoring the dehydrogenase activity. Data represent the mean of triplicate experiments with error bars representing standard deviations

$c s i D$ operon genes is induced. CsiR is homologous to the gluconate repressor ${ }^{30}$ and hence predicted to be ligand-dependent. We purified CsiR from E. coli and utilizing a filter binding assay we demonstrate that the repressive effect at the csiD promoter site is specifically relieved upon GA binding, with related compounds showing no or less ( $\alpha \mathrm{KG}$ ) effects (Fig. 5b, Supplementary Figure 12). We further examined the DNA binding site upstream of csiD by hydroxyl radical footprinting (Supplementary Figure 13a, b) ${ }^{31}$. We found that CsiR binds to two primary and two secondary sites in the promoter region of the $c s i D$ operon with the

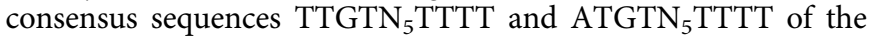
primary sites, each separated by six nucleotides (Fig. 5c). Surface plasmon resonance studies determined a dissociation constant $K_{\mathrm{d}}$ $=10 \mathrm{nM}$ for the CsiR/DNA interaction (Supplementary Figure $14 \mathrm{a}, \mathrm{b})$.

\section{Discussion}

Lysine is originally thought to be degraded in a ketogenic manner via $\beta$-oxidation and the formation of acetyl-CoA. In accordance with that, GA is an intermediate of lysine degradation in Pseudomonads and is proposed to be converted to glutaryl-CoA which is further metabolized to acetyl-CoA ${ }^{32}$. Recently, a study of lysine degradation in P. putida KT2440 was published that identified and characterized the same pathway via CsiD and LhgO as in this work ${ }^{33}$. The authors also demonstrated that $E$. colipossesses the key enzymes CsiD and LhgO necessary to degrade lysine via GA and L2HG. Hence, in E. coli, lysine is metabolised in a glucogenic way by the conversion of GA to SA and $\mathrm{CO}_{2}$ via aKG-dependent hydroxylation and subsequent oxidation to aKG. This pathway fills a gap in central carbon and energy metabolism. In $E$. coli, the pathway is activated in the stationary phase in a cAMP/CRP-dependent manner. Hence, it seems that lysine is recycled by $E$. coli in stationary phase for carbon and energy regeneration. The described regulation is in accordance with growth experiments with the $\Delta c s i D$ strain exhibiting a growth defect at the onset of the stationary phase compared to WT E. coli K-12 (Supplementary Figure 15), hinting at a distinct advantage of activating lysine degradation via the $c s i D$ pathway in the stationary phase. Apart from introducing reactions of central metabolites, our findings have implications for the biotechnological production of the polyamide building blocks AVA and GA. Both compounds have been produced from engineered $E$. coli before ${ }^{34,35}$. Taking into account the presented pathways and activities, it is likely that yields could be improved significantly by utilizing knockout strains or adopting conditions such as oxygen limitation preventing degradation of the target compounds by GabT/D and CsiD/LhgO. Indeed, Zhang and coworkers demonstrate increased production of GA by knocking out both the glutarate hydroxylation as well as the glutaryl-CoA dehydrogenase pathway in $P$. putida ${ }^{33}$.

Furthermore, the presented pathway has potential implications for diseases such as cancer ${ }^{9}$ and genetic organic acidurias ${ }^{36}$. In humans, L2HG is produced by malate and lactate dehydrogenases especially at acidic conditions caused e.g., by hypoxia ${ }^{37}$. Increased L2HG levels are malignant due to inhibition of TET-type and Jmjc-type aKG-dependent oxygenases responsible for nucleobase and histone demethylation, resulting in epigenetic deregulation of gene expression and thereby progression in certain cancers ${ }^{13,14}$. Some tumours are also more prevalent in genetic acidurias defined by elevated levels of $\mathrm{L} 2 \mathrm{HG}^{38}$. Genetic inactivation of L2HGDH (the human homologue of LhgO) is the cause of $L$ hydroxyglutaric aciduria ${ }^{39}$. Increased GA levels are found in glutaric aciduria type $\mathrm{I}^{40}$ and glutaric aciduria type $\mathrm{III}^{41}$. The glutaric acidurias are caused by malfunctioning glutaryl-CoA dehydrogenase and succinyl-CoA/glutaryl-CoA transferase, respectively ${ }^{41}$. However, the source of free GA in human metabolism is not fully understood since interrupting the saccharopine pathway upstream of glutaryl-CoA formation in a mouse model for GA-I did not rescue the mice from developing the disease ${ }^{42}$. Moreover, certain cases of glutaric aciduria responded to antibiotics treatment, hinting at the possibility that gut bacteria could contribute to elevated levels of $\mathrm{GA}^{43}$. Interestingly, questions regarding additional sources of GA and hydroxyglutarates and their potential involvement in normal metabolic processes have been raised frequently 9 . In this regard, the presented work is potentially relevant for the discussed diseases and could inspire further research identifying sources and fates of GA and L2HG in humans.

\section{Methods}

Enzyme nomenclature. We suggest that the discovered glutarate hydroxylase belongs to subgroup EC 1.14.11. with the name glutarate-2-hydroxylase (G-2-H) 


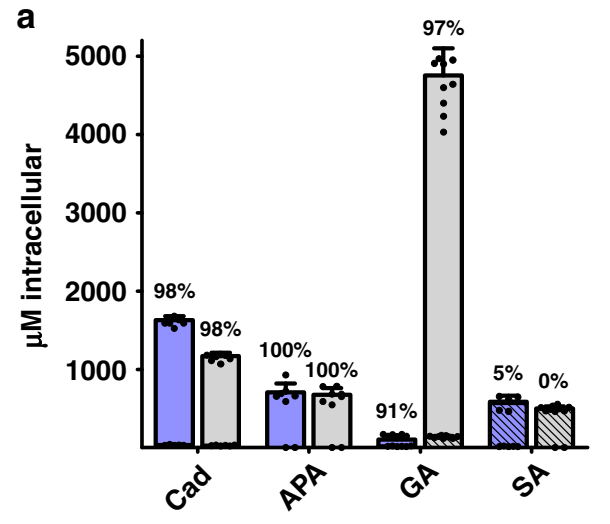

b
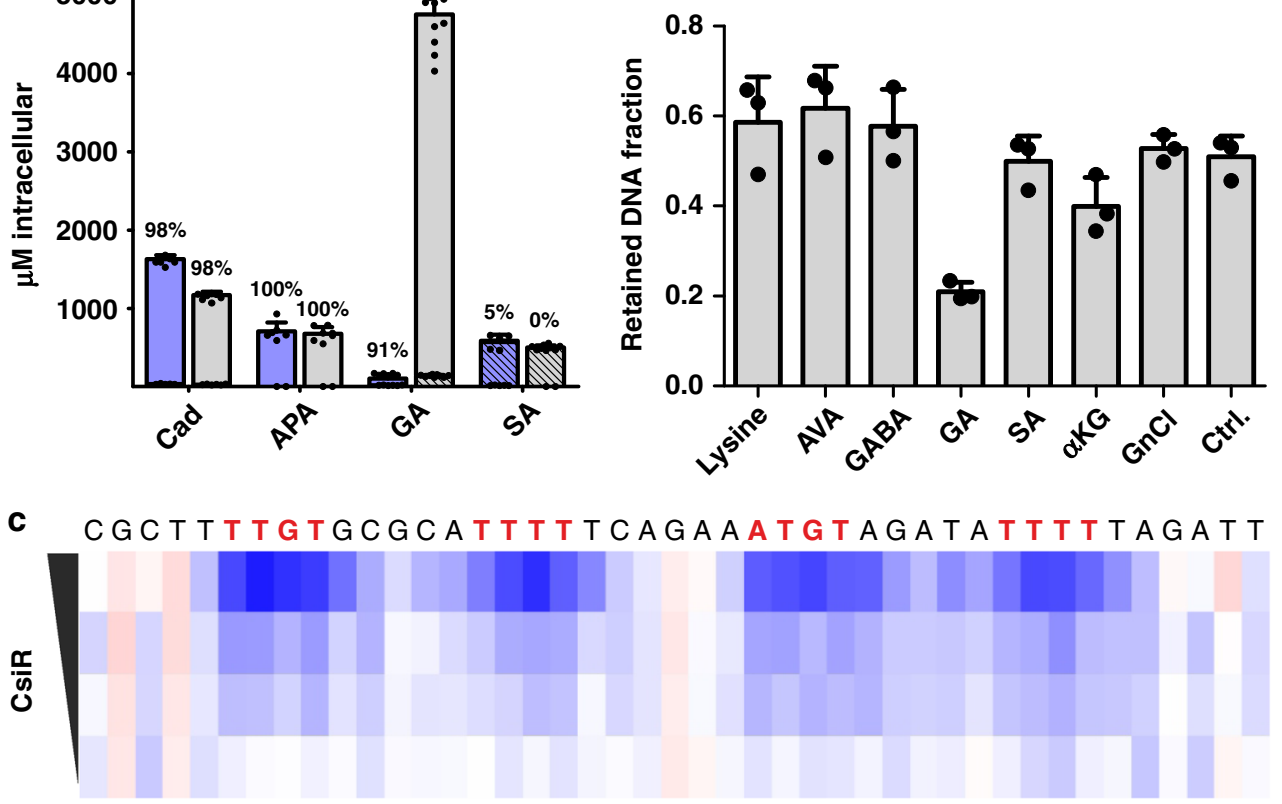

Fig. 5 Isotope tracing and regulation of the lysine degradative pathway in E. coli. a Intracellular concentrations of cadaverine (Cad), 5-aminopentanal (APA), glutarate (GA) and succinate (SA) of E. coli K-12 wt (blue) and $\Delta c s i D$ (grey) in stationary phase cultures. Non-isotope-labelled compounds are indicated as shaded bars. The sum of ${ }^{13} \mathrm{C}$-labeled metabolites are shown as blank bars. Fractions of labelled compounds relative to the sum of labelled and non-labelled compounds are indicated above the respective bar (in percent). For isotope labelling patterns see Supplementary Table 3. Data represent the means of biological triplicates with error bars representing standard deviations. Each replicate was measured at least once. $\mathbf{b}$ characterisation of the allosteric repressor CsiR of the csiD operon: Ligand specificity of CsiR repression determined by a filter binding assay (Supplementary Figure 8) of CsiR and a dsDNA sequence derived from the csiD operon promoter region in presence of annotated compounds ( $1 \mathrm{mM}$ each, GnCl: Guanidiniumchloride, Ctrl: no ligand). CsiR-bound radioactive DNA is retained on a nitrocellulose membrane. GA specifically reduces binding of CsiR to the DNA. and $\mathbf{c}$ determination of dsDNA consensus motif recognized by CsiR determined by hydroxyl footprinting (Supplementary Figure 13a, b). Increasing CsiR concentrations protect DNA from hydroxyl radical cleavage (decreased cleavage is visualised in blue)

and systematic name glutarate, 2-oxoglutarate:oxygen oxidoreductase ((2S)hydroxylating). We propose to rename the csiD gene to glutarate hydroxylase $(\mathrm{glaH})$. LhgO should be reclassified as L-2-hydroxyglutarate dehydrogenase belonging to EC 1.1.99.2 (systematic name: (S)-2-hydroxyglutarate:quinone-oxidoreductase) instead of 1.1.3.15 ((S)-2-hydroxy-acid oxidase) and the gene should be renamed from $l h g O$ to $\ln g D$ in analogy to the homologous dehydrogenases. The gene encoding the HTH-type transcriptional repressor could be renamed from $c s i R$ to glaR.

Bacterial strains and general culture conditions. Escherichia coli BW25113 and its single gene knockout derivatives $\Delta c s i D, \Delta g a b T, \Delta g l c D$, and $\Delta l h g O$ from the Keio collection $^{44}$, that were used for analysis, were all obtained from the National BioResearch Project (NIG, Japan): E. coli. Genes encoding CsiD, CsiR, GabD, GabT, LhgO, PuuE, Sad were amplified and restriction enzyme sites were introduced with primers listed in Supplementary Table 4. Escherichia coli BW25113 was used as template. PCR fragments were inserted into vector pET28a $\left(\mathrm{Km}^{\mathrm{r}}\right)$ carrying a T7-promoter under control of lacI using standard protocols. Thus, overexpression vectors were created for CsiD, CsiR, GabD, GabT, PuuE, and Sad ORFs carrying a $6 \times \mathrm{N}$-terminal His-tag and for LhgO carrying a $6 \times \mathrm{C}$-terminal His-tag. To create a complemented $l h g O+$ strain, $l h g O$ was inserted into pET16b using restriction sites and primers listed in Supplementary Table 4. Recombinant cloning vectors were transformed into Escherichia coli BL21 (DE3) (Stratagene, USA, La Jolla) via electroporation. Plasmid constructs were validated by sequencing using primers SP04 and SP10. Bacterial strains were either grown in LB medium or M9 minimal medium containing $8.5 \mathrm{~g} / \mathrm{l} \mathrm{Na}_{2} \mathrm{HPO}_{4} \cdot 2 \mathrm{H}_{2} \mathrm{O}, 3 \mathrm{~g} / \mathrm{KH}_{2} \mathrm{PO}_{4}, 0.5 \mathrm{~g} / \mathrm{l}$ $\mathrm{NaCl}, 1 \mathrm{~g} / \mathrm{l} \mathrm{NH}_{4} \mathrm{Cl}, 2 \mathrm{mM} \mathrm{MgCl}_{2}, 100 \mu \mathrm{M} \mathrm{CaCl}_{2}$ supplemented with trace elements $\left(0.1 \mathrm{mM}\right.$ EDTA, $0.03 \mathrm{mM} \mathrm{FeCl}_{3}, 6.2 \mu \mathrm{M} \mathrm{ZnCl}_{2}, 0.76 \mu \mathrm{M} \mathrm{CuCl}_{2}, 0.42 \mu \mathrm{M} \mathrm{CoCl}_{2}$, $1.62 \mu \mathrm{M} \mathrm{H}_{3} \mathrm{BO}_{3} ; 0.08 \mu \mathrm{M} \mathrm{MnCl}_{2}$ ) and vitamins $(0.1 \mathrm{mg} / \mathrm{l}$ cyanocobalamin, $0.08 \mathrm{mg} /$ 14 -aminobenzoic acid, $0.02 \mathrm{mg} / \mathrm{l} \mathrm{D}$-(+)-biotin, $0.2 \mathrm{mg} / \mathrm{l}$ niacin, $0.1 \mathrm{mg} / \mathrm{l} \mathrm{Ca-D}$ (+)-pantothenic acid, $0.3 \mathrm{mg} / \mathrm{l}$ pyridoxamine-chloride, $0.2 \mathrm{mg} / \mathrm{l}$ thiamindichloride) at $37^{\circ} \mathrm{C}$. As carbon source in minimal medium $0.2 \%(\mathrm{w} / \mathrm{v})$ glucose was used. When necessary, medium was supplemented with $30 \mu \mathrm{g} / \mathrm{ml}$ kanamycin.

Growth on different C-sources and $\mathbf{N}$-sources. E.coli strains were grown in 96 deep well plates in minimal medium as triplicates containing trace elements supplemented with $10 \mathrm{mM} \mathrm{C}$-sources (lysine, cadaverine, 5-aminovalerate, glutarate) or N-Sources (lysine, cadaverine, 5-amniovalerate). Growth was monitored by measuring $\mathrm{OD}_{600}$ in 96 well plates by Tecan plate reader. Growth (means of triplicates) was rated as high growth $\left(0.47>\mathrm{OD}_{600}>0.43\right.$ a.u. $)$; intermediate growth $\left(0.35>\mathrm{OD}_{600}>0.25\right.$ a.u. $)$, low growth $\left(0.15>\mathrm{OD}_{600}>0.07\right.$ a.u. $)$ or no growth $\left(0.02\right.$ a.u $\left.>\mathrm{OD}_{600}\right)$.

Protein purification. Strains were grown in $\mathrm{LB}$ with suitable antibiotic to an $\mathrm{OD}_{600}$ of 0.5. Protein expression was induced with $1 \mathrm{mM}$ IPTG and cells were grown for $4-5 \mathrm{~h}$. Cells were harvested by centrifugation at $4000 \mathrm{rpm}$ at $4{ }^{\circ} \mathrm{C}$. Cells were lysed by sonication in lysis buffer $\left(50 \mathrm{mM} \mathrm{NaH} \mathrm{PO}_{4}, 300 \mathrm{mM} \mathrm{NaCl}, 10 \mathrm{mM}\right.$ imidazole) with Branson sonifier for 3 min duty cycle at $20 \%$ power with 0.5 on and $0.5 \mathrm{~s}$ off Cell debris was removed by centrifugation at $10,000 \mathrm{rpm}$ at $4{ }^{\circ} \mathrm{C}$ and the cell lysate incubated with Ni-NTA beads (Qiagen) for $1 \mathrm{~h}$ at $4^{\circ} \mathrm{C}$ at a rotary shaker. Beads were washed twice with wash buffer $\left(50 \mathrm{mM} \mathrm{NaH}_{2} \mathrm{PO}_{4}, 300 \mathrm{mM} \mathrm{NaCl}, 20 \mathrm{mM}\right.$ Imidazole) and the protein finally eluted with elution buffer $\left(50 \mathrm{mM} \mathrm{NaH} \mathrm{NO}_{4}\right.$, $300 \mathrm{mM} \mathrm{NaCl}, 500 \mathrm{mM}$ Imidazole). Purity was checked by SDS-gel. Protein was transferred to the respective buffer system required for the experiment by size exclusion chromatography (PDE-10 columns, GE healthcare).

NMR of the CsiD reaction. The reaction of $\alpha$-ketoglutarate $(10 \mathrm{mM})$ and glutarate $(10 \mathrm{mM})$ catalysed by CsiD in $20 \mathrm{mM}$ ammonium acetate buffer $\mathrm{pH} 7.25$ was monitored by ${ }^{1} \mathrm{H}$ NMR spectra without purification. ${ }^{1} \mathrm{H}$ NMR spectra were recorded at $300 \mathrm{~K}$ on Bruker Avance III $400 \mathrm{MHz}$ spectrometers (ayita 400 and isa 400 ) with a BBFO plus probe for $\mathrm{N}$ to $\mathrm{F} / \mathrm{H}$ or $\mathrm{F}$. Data for NMR spectra were recorded as follows: chemical shift $(\delta, \mathrm{ppm})$, multiplicity (s, singlet; $\mathrm{d}$, doublet; $\mathrm{t}$, triplet; q, quartet; m, multiplet), integration, coupling constant (Hz). Acquired data was processed and analysed using MestReNova software.

CsiD reactions analysed by Clark-type $\mathbf{O}_{\mathbf{2}}$ electrode. Reactions were conducted in $100 \mathrm{mM}$ MOPS, $70 \mathrm{mM} \mathrm{NaCl}$ and $20 \mathrm{mM} \mathrm{KCl} \mathrm{pH} 7.2$ in the presence of $400 \mu \mathrm{M}$ Ascorbat and $4 \mu \mathrm{M} \mathrm{Fe}\left(\mathrm{NH}_{4}\right)_{2} \mathrm{SO}_{4}$. $K_{\mathrm{M}}$ for glutarate and $\alpha$-ketoglutarate was determined in the presence of $1 \mathrm{mM}$ a-ketoglutarate and $5 \mathrm{mM}$ glutarate, respectively. Five millimolar substrate was used for the $\mathrm{C} 2-\mathrm{C} 7$ analogues of glutarate. 180 $\mathrm{nM}$ CsiD were used for the reactions. Reactions were conducted at $30^{\circ} \mathrm{C}$ in the sealed reaction chamber with magnetic stirrer. $v_{0}$ was determined and plotted 
against the concentration. The plot was fitted with Michaelis-Menten equation with in GraphPad Prism5 (HYPNOS).

\section{Stereospecificity of the CsiD reaction. Evaluation of the 2-hydroxyglutarate} enantiomer was performed as described before ${ }^{12}$. The CsiD reaction was performed as before (oxygen electrode) for $1 \mathrm{~h}$ at $30^{\circ} \mathrm{C}$. Reaction was incubated for 10 min at $70^{\circ} \mathrm{C}$ to heat-inactivate $\mathrm{CsiD}$. Denatured protein was removed by centrifugation $(14,000 \mathrm{rpm})$. Supernatant of the CsiD reaction and $L-2$ -

hydroxyglutarate and $D$-hydroxyglutarate as controls were derivatised with $50 \mathrm{~g} / \mathrm{L}$ diacetyl- $L$-tartaric anhydride in $4: 1(\mathrm{v} / \mathrm{v})$ dichloromethane/acetic acid for $30 \mathrm{~min}$ at $75^{\circ} \mathrm{C}$. Supernatant was evaporated to dryness and residue was dissolved in water. Derivatised products were analysed by LC-MS and identified by comparison to standards.

Crystallisation of CsiD-ligand complexes. Purified CsiD protein was concentrated to $13 \mathrm{mg} / \mathrm{mL}$ and its crystallization in liganded form pursued by cocrystallization, crystal soaking or a combination of both approaches, as follows:

Apo CsiD: crystals grew from reservoir solutions containing $80 \mathrm{mM}$ sodium chloride, $12 \mathrm{mM}$ potassium chloride, $20 \mathrm{mM}$ magnesium chloride hexahydrate, 40 $\mathrm{mM}$ sodium cacodylate trihydrate $\mathrm{pH}$ 6.0, 30\% [v/v] 2-methyl-2,4-pentanediol, 12 $\mathrm{mM}$ spermine tetrahydrochloride.

CsiD-GA: crystals from apo CsiD (obtained as described in i) were soaked in 10 $\mathrm{mM}$ glutarate prior to flash cooling.

CsiD-SA: CsiD was co-crystallised with succinate by using a mother liquor containing $1.0 \mathrm{M}$ succinic acid $\mathrm{pH} 7.0,0.1 \mathrm{M}$ Bis-Tris propane $\mathrm{pH} 7.0$

CsiD-NOG: CsiD protein was incubated with NOG at a CsiD:NOG molar ratio of $1: 5$ for $1 \mathrm{~h}$ on ice and the mixture used in crystallisation trials. Crystals grew from 1.0 M magnesium sulfate, $0.1 \mathrm{M}$ Tris $\mathrm{pH}$ 8.5. Prior to flash freezing, crystals were soaked in $20 \mathrm{mM}$ NOG to improve ligand occupancy.

In all cases, crystallization was performed in 96-well Intelliplates (Art Robbins) and used the sitting drop, vapour diffusion format implemented with a Gryphon (Art Robbins) nanolitre dispensing robot. Drops consisted of $200 \mathrm{~nL}$ protein or protein/ligand mixture and $200 \mathrm{~nL}$ reservoir solution, with reservoirs containing $70 \mu \mathrm{L}$ mother liquor. All crystallization trials were incubated at $18^{\circ} \mathrm{C}$. For X-ray data collection, crystals were harvested into LithoLoops (Hampton Research) and cryo-protected in mother liquor supplemented with $20 \%$ [v/v] glycerol.

\section{X-ray data collection and structure elucidation. X-ray diffraction data were} collected at beamline PXI (X06SA) of the Swiss Light Source synchrotron (Villigen, Switzerland) equipped with an Eiger $16 \mathrm{M}$ detector (Dectris, Switzerland). Data were collected at a wavelength of $1.00 \AA$ with $0.1^{\circ}-0.2^{\circ}$ oscillation per frame. Data processing used the XDS/XSCALE package ${ }^{45}$. Phasing was performed by molecular replacement in $\mathrm{PHASER}^{46}$. First, a CsiD protomer obtained from PDB entry $2 \mathrm{R} \mathrm{S}^{15}[10.2210 / \mathrm{pdb} 2 \mathrm{R} 6 \mathrm{~S} / \mathrm{pdb}]$ was used as search model for the elucidation of the apo CsiD structure in this work. The resulting apo model was then used for the phasing of liganded crystal forms of CsiD. Model refinement used phenix.refine ${ }^{47}$ and manual model building was performed in $\mathrm{COOT}^{48}$. Ligand restraints were generated using ELBOW ${ }^{49}$. Non-crystallographic symmetry restraints and TLS refinement were applied as part of model refinement in phenix.refine ${ }^{47}$. All structures had good Ramachandran values (Favoured: >98\%; Disallowed: 0\%). Xray data statistics and model parameters are given in Supplementary Table 1. (Diffraction data and model coordinates have been deposited with the Protein Data Bank. Accession codes are given in Supplementary Table 1).

Kinetics of GabT/D and Sad/PuuE and PatA/D. Assays were carried out in a buffer $(\mathrm{pH}=8.0)$ containing $5 \mathrm{mM}$ a-ketoglutarate, $500 \mu \mathrm{M} \mathrm{NADP}^{+}(\mathrm{GabT} / \mathrm{D}$ \& $\mathrm{Sad} / \mathrm{PuuE}$ ) or $\mathrm{NAD}^{+}$(PatA/D), $100 \mu \mathrm{M}$ DTT, $100 \mathrm{mM}$ sodiumpyrophosphat and $0.01 \mathrm{mg} / \mathrm{mL}$ of both purified GabT/D, Sad/PuuE or PatA/D. Reactions were conducted at room temperature and started by the addition of different concentrations of AVA or GABA (GabT/D \& Sad/PuuE) respectively cadaverine or putrescine (PatA/D). Increase of NADPH or NADH was monitored over time measuring the absorbance at $340 \mathrm{~nm}$, thus determining the kinetic of the coupled enzyme reaction. The reduction of $\mathrm{NADP}^{+}$to $\mathrm{NADPH}$ corresponds stoichiometrically to the conversion of GABA (or AVA) to succinate semialdehyde (or glutarate semialdehyde) and then to succinate (or glutarate) (GabT/D). In case of PatA/D, NAD ${ }^{+}$ reduction corresponds to the conversion of putrescine (or cadaverine) to aminobutanal (or aminopentanal) followed by oxidation to GABA (or AVA) catalysed by PatD. Decoupling the assay by first starting the transaminase reaction followed by addition of the dehydrogenase did not alter overall velocities concluding that the dehydrogenase reaction must be the rate limiting step in the reaction. Starting velocities $\left(v_{0}\right)$ of the reaction were plotted against substrate concentrations and non-linear regressions were calculated with GraphPad Prism 5 (HYPNOS).

Preparation of $\boldsymbol{E}$. coli membrane fractions. Wildtype and knockout E. coli strains were inoculated in $200 \mathrm{~mL} \mathrm{LB}$ medium and incubated over night at $37^{\circ} \mathrm{C}$ at 200 $\mathrm{rpm}$ on a rotary shaker. Overnight culture was centrifuged and the pellet was washed twice with ice-cold membrane fraction buffer containing $50 \mathrm{mM}$ HEPES, $10 \mathrm{mM}$ potassiumacetate, $10 \mathrm{M} \mathrm{CaCl}_{2}, 5 \mathrm{mM} \mathrm{MgCl}_{2}$ titrated with $\mathrm{NaOH}$ to $\mathrm{pH}=$ 7.5 and resuspended in $10 \mathrm{~mL}$ buffer. All following steps were carried out on ice or at $4{ }^{\circ} \mathrm{C}$. Cell suspension was passed four times through a french press cell at 10,000 psi. To remove unlysed cells and insoluble cell debris the lysate was centrifuged at $20,000 \times g$ for $20 \mathrm{~min}$. Supernatant was centrifuged for $30 \mathrm{~min}$ at $100,000 \times g$. After ultracentrifugation supernatant was stored as cytosolic fraction and the brownish membrane pellet was washed with membrane fraction buffer $(5 \mathrm{~mL})$ and centrifuged again. The membrane pellet was resuspended in $500 \mu \mathrm{L}$ membrane fraction buffer. Protein concentration of cytosolic and membrane fraction was determined via BSA-Bradford assay.

Activity of purified LhgO and membrane fractions. Prior to analysis a PD midiTrap G-25 column (GE Healthcare) was used to exchange buffer of purified LhgO to retain the protein in reaction buffer containing $25 \mathrm{mM}$ HEPES, $100 \mathrm{mM}$ $\mathrm{NaCl}, 5 \mathrm{mM}$ EDTA ( $\mathrm{pH}=7.5$ ). LhgO activity was assayed spectrophotochemically in reaction buffer. Direct reduction of $100 \mu \mathrm{M}$ ubiquinone-1 ( $\mathrm{UQ}_{1}$ ) (coenzyme Q1, Sigma Aldrich Co.) by purified LhgO was measured by the decrease of absorbance at $278 \mathrm{~nm}$. A final enzyme concentration of $650 \mathrm{nM}$ was used and the reaction was monitored dependent on L-2-hydroxyglutarate concentration. Differential absorption coefficient of $\mathrm{UQ}_{1}$ at this wavelength in reaction buffer was determined as $\Delta \varepsilon=8.36 \mathrm{mM}$. Enzyme activities were determined as $\mathrm{UQ}_{1}$ reduced per minute. Specific activities were referred to $0.03 \mathrm{mg} / \mathrm{mL} \mathrm{LhgO} \mathrm{used} \mathrm{in} \mathrm{the} \mathrm{assay.} \mathrm{To} \mathrm{exclude}$ possible effects of $\mathrm{H}_{2} \mathrm{O}_{2}$ produced by $\mathrm{LhgO}$ on redox dyes $100 \mathrm{U} / \mathrm{mL}$ catalase (Catalase from bovine liver, Sigma Aldrich Co.) was added. Initial velocities $\left(v_{0}\right)$ of the reaction were plotted against L2HG concentrations and non-linear regressions were calculated with GraphPad Prism 5 (HYPNOS).

Redox activity of $0.1 \mathrm{mg} / \mathrm{mL}$ total protein of membrane-fraction and cytosolicfraction was determined in membrane fraction buffer $(50 \mathrm{mM}$ HEPES, $10 \mathrm{mM}$ potassiumacetate, $\left.10 \mathrm{mM} \mathrm{CaCl}_{2}, 5 \mathrm{mM} \mathrm{MgCl}_{2}, \mathrm{pH}=7.5\right)$. Reduction of $100 \mu \mathrm{M}$ DCPIP by purified LhgO $(217 \mathrm{nM})$ or membrane-bound enzyme was monitored in presence or absence of $50 \mu \mathrm{M}$ ubiquinone and menaquinone-4 $\left(\mathrm{MQ}_{4}\right)$ (menaquinone K2, Sigma Aldrich Co.) by absorbance measurement at $600 \mathrm{~nm}$. Oxidized DCPIP has an absorbance maximum at $600 \mathrm{~nm}$. Activities of membranebound and purified LhgO were determined as DCPIP reduced per minute (nmol/ min). DCPIP reduction of $E$. coli WT membranes compared to wildtype cytosolic fraction and membranes isolated from a $\Delta l h g O$ strain were conducted in the presence of $50 \mu \mathrm{M} \mathrm{UQ}_{1}$. DCPIP reduction assays were carried out at room temperature and started by the addition of $5 \mathrm{mM} \mathrm{L}$-2-hydroxyglutarate.

Oxygen consumption by membranes and purified LhgO were measured in a Clark-type electrode at room temperature. Activity of membrane-bound LhgO upon titration of the respiratory chain inhibitor HQNO was determined by oxygen consumption and DCPIP reduction.

Bacterial ubiquinone reduction. Membrane fractions of E.coli WT and $\Delta l h g O$ were prepared as described in the section "Preparation of E. coli membrane fractions". In brief, cells were lysed in a french press and membranes were isolated by ultracentrifugation. Total protein concentration was determined. One hundred and fifty microgram of the membrane fraction in $200 \mu \mathrm{L}$ membrane fraction buffer (50 mM HEPES, $10 \mathrm{mM}$ potassiumacetate, $10 \mathrm{mM} \mathrm{CaCl}_{2}, 5 \mathrm{mM} \mathrm{MgCl}_{2}, \mathrm{pH}=7.5$ ) were incubated for $1 \mathrm{~h}$ at $25^{\circ} \mathrm{C}$ under nitrogen atmosphere in the presence or absence of $5 \mathrm{mM}$ L2HG or SA. Quinones were extracted with $1 \mathrm{~mL}$ of a 1:3:1 mixture of methanol:hexane:acetone. Samples were dried under a constant flow of nitrogen and analysed by HPLC coupled to a PDA detector (Shimadzu) on a Eurospher RP C18 column $(125 \times 3 \mathrm{~mm}$; Knauer $)$. Mobile phases contained $100 \%$ methanol (A) and $10 \%$ heptane in methanol (B). Isocratic elution with $100 \% \mathrm{~A}$ was performed for $10 \mathrm{~min}$ followed by a linear gradient from 0 to $50 \% \mathrm{~B}$ in $2 \mathrm{~min}$ and 50 to $100 \%$ B in $12 \mathrm{~min}$. Spectra were recorded from $200 \mathrm{~nm}$ to $400 \mathrm{~nm}$. Area under the curve were determined for ubiquinol (RT: $9 \mathrm{~min} ; 290 \pm 4 \mathrm{~nm}$ ) and ubiquinone (RT $16 \mathrm{~min} ; 275 \mathrm{~nm} \pm 4 \mathrm{~nm}$ ). Statistical significance of the data was assessed by an unpaired one-tailed Student's $t$-test in GraphPad Prism5 (HYPNOS). The nature of the quinone species and reduction state was confirmed by the corresponding retention times and absorption spectra ${ }^{50-52}$. Furthermore, ubiquinone from the membrane fractions could be assigned to the mass of ubiquinone- 8 by mass spectrometry.

Growth experiments with isotopically labelled lysine. For growth and metabolite analysis of Escherichia coli BW25113 WT and $\Delta c s i D$ cells were inoculated in $100 \mathrm{~mL}$ M9 minimal medium supplemented with glucose in the presence of $10 \mathrm{mM}$ lysine in $500 \mathrm{~mL}$ baffled flasks. Cultures were grown in duplicates at $37^{\circ} \mathrm{C}$ on a rotary shaker at $200 \mathrm{rpm}$. Cell growth over time was determined spectrophotochemically by measuring optical density at $600 \mathrm{~nm}$ in a $1 \mathrm{~mL}$ cuvette at indicated intervals. For monitoring intracellular metabolite concentrations during E. coli culture growth samples with a volume equivalent to $\mathrm{OD}_{600}=0.1 / \mathrm{mL}$ were taken at indicated time points.

For ${ }^{13} \mathrm{C}$ labeling experiments WT and $\Delta c s i D$ cells were inoculated in triplicates supplemented with $10 \mathrm{mM}$ L-lysine- ${ }^{13} \mathrm{C}_{6},{ }^{15} \mathrm{~N}_{2}$ hydrochloride (Sigma Aldrich Co.) in $5 \mathrm{~mL}$ M9 minimal medium containing $0.2 \%(\mathrm{w} / \mathrm{v})$ glucose in $50 \mathrm{~mL}$ falcons and incubated at $37^{\circ} \mathrm{C}$ for $24 \mathrm{~h}$ at $200 \mathrm{rpm}$. After $24 \mathrm{~h}$ a volume equivalent to $\mathrm{OD}_{600}=$ $0.2 / \mathrm{mL}$ was harvested and centrifuged at $-9^{\circ} \mathrm{C}$. To rapidly quench metabolic activities the harvested cells were methanol-extracted by resuspending the cell pellet in $2 \mathrm{~mL} 80 \%(\mathrm{v} / \mathrm{v})$ methanol cooled down close to freezing with liquid 
nitrogen. The suspension was frozen in liquid nitrogen for $1 \mathrm{~min}$, thawed on ice, centrifuged for $10 \mathrm{~min}$ at $10,000 \times g$ and the supernatant was stored as metabolite extract. This step was repeated three times. The supernatant fractions were pooled and lyophilized to dryness. Lyophilized metabolite extract was dissolved in $500 \mu \mathrm{L}$ deionized water and analyzed by LC-MS.

Analysis of lysine degradation metabolites by LC-MS. Identification of metabolites were performed with UltiMate 3000 HPLC system and LTQ Orbitrap Velos (Thermo Scientific). Nucleodur C18 ISIS column $(250 \mathrm{~mm}$ length x $2 \mathrm{~mm}$ i.d., 2.7 $\mu \mathrm{m}$ particle size, Macherey-Nagel, Germany, Düren) was used. The injection volume was $10 \mu \mathrm{L}$ and the flow rate was $0.25 \mathrm{~mL} \mathrm{~min}^{-1}$. The mobile phases contained $10 \mathrm{mM}$ ammonium formate ( $\mathrm{pH} 3.2)(\mathrm{A})$ and $0.1 \%$ formic acid in acetonitrile (B). The linear gradient comprised 0 to $30 \%$ B for $10 \mathrm{~min}$ and $90 \%$ B for 2 min. The MS scan ranged from an $m / z 100-400$ with a resolution of 100,000 at $\mathrm{m} / \mathrm{z}$ 400 was achieved in positive and negative ionization modes. Accurate mass $( \pm 3$ $\mathrm{ppm})$ and retention time $( \pm 0.2 \mathrm{~min})$ values were used for molecular assignment. For the analysis of hydroxyglutarate derivatised with DATAN Prominence HPLC system with LCMS-2020 single quadrupole MS (Shimadzu) was applied. HPLC conditions was as previously described. MS Detection was performed in single ion monitoring (SIM) negative ionization mode at $\mathrm{m} / \mathrm{z}$ 363. The same instrumentation was used for the quantification of metabolites in cell extracts. Prior to analysis 16 $\mu \mathrm{L}$ of aqueous metabolite extract was mixed with $8 \mu \mathrm{L}$ phase B ( $90 \%$ acetonitrile, $0.2 \%$ formic acid, $10 \mathrm{mM}$ ammonium formate), $5 \mu \mathrm{L}$ of this solution was injected. Metabolites were separated using Nucleodur HILIC column $(250 \mathrm{~mm}$ length $\mathrm{x} 2$ $\mathrm{mm}$ i.d., $3 \mu \mathrm{m}$ particle size, Macherey-Nagel), which was equilibrated with buffer B and eluted with a linear gradient of $45 \%$ buffer A ( $10 \mathrm{mM}$ ammonium formate, $\mathrm{pH}$ 3.0) over a 10-min period followed by isocratic step of $45 \%$ buffer B for $8 \mathrm{~min}$. Column was operated at $(35.0 \pm 0.1)^{\circ} \mathrm{C}$ with a flow rate of $0.15 \mathrm{ml} / \mathrm{min}$. SIM detection of corresponding protonated ions in positive ionization mode was used for lysine, cadaverine and 5-aminovalerate. Glutarate, succinate and $a$ ketoglutarate ions were detected in negative SIM mode. Ions used for identification and quantification of compounds by LC-MS are summarised in Supplementary Table 2 .

To identify and quantify compounds in cell extracts standard solutions of pure substances were measured under the same conditions. Calibration curves for cadaverine, glutarate and succinate were obtained for the measuring range and used for quantification of these substances. Intracellular metabolite concentrations were calculated according to following equation:

$$
C_{\text {avg }}=C_{\text {ex }} \times V_{\text {ex }} \times \frac{\mathrm{DW}_{\text {cell }}}{\mathrm{DW}_{\text {tot }} \times V_{\text {cell }}},
$$

with $C_{\text {ex }}$ being the metabolite concentration of the extraction solution determined via external calibration. $V_{\mathrm{ex}}$ is the volume of the extraction solution $(0.5 \mathrm{~mL})$. $\mathrm{DW}_{\text {tot }}$ is the experimentally determined total dry weight of the metabolite sample. $\mathrm{DW}_{\text {cell }}$ is the dry weight per cell $\left(3 \times 10^{-13} \mathrm{~g}\right)$ and $V_{\text {cell }}$ is the volume per cell $(6.7 \times$ $\left.10^{-16} \mathrm{~L}\right)^{53}$

Filter binding of CsiR/dsDNA interaction. Primer MS161 was $5^{\prime}$-labelled with $\gamma$ P32-ATP (Hartmann Analytics) by T4 PNK (NEB). PCR was conducted with labelled MS161 and MS162 on genomic DNA from E. coli K-12 MG1655. PCR product was purified by agarose gel extraction. DNA $(500 \mathrm{cps})$ was incubated for $15 \mathrm{~min}$ in the presence of purified CsiR and $1 \mathrm{mM}$ compound (as assigned) in binding buffer $\left(50 \mathrm{mM}\right.$ Tris- $\mathrm{HCl}, 100 \mathrm{mM} \mathrm{KCl}, 50 \mathrm{mM} \mathrm{NaCl}, 5 \mathrm{mM} \mathrm{MgCl}_{2} \mathrm{pH}=$ 7.6). Reaction was dot-blotted on nitrocellulose membrane $(0.2 \mu \mathrm{M}$, Roth) followed by a nylon membrane $(0.45 \mu \mathrm{M}$, Roth). Binding was determined by the ratio of bound DNA (Intensity on NC membrane) to entire DNA (Sum of Intensity on NC membrane and nylon membrane). Radiograph was recorded with Thypoon FLA 7000 (GE Healthcare).

Hydroxyl radical footprint. Primer MS170 was 5'-labelled with $\gamma$-P32-ATP (Hartmann Analytics) by T4 PNK (NEB). PCR was conducted with labelled MS170 and MS171 on genomic DNA from E. coli K-12 MG1655. Binding of CsiR was allowed for $15 \mathrm{~min}$ at $25^{\circ} \mathrm{C}$ in $50 \mathrm{mM}$ MOPS, $100 \mathrm{mM} \mathrm{NaCl}, 20 \mathrm{mM} \mathrm{KCl}, 2.5 \mathrm{mM}$ $\mathrm{MgCl}_{2} 0.1 \mathrm{mM}$ DTT $\mathrm{pH}=7.2$. Footprint reaction was performed as described before $^{31}$. Additionally, a G specific Maxam-Gilbert sequencing reaction was performed as a control to assign the nucleotides. DNA fragments were separated on a $10 \%$ denaturing PAGE. Radiograph was recorded with Thypoon FLA 7000 (GE Healthcare). The radiograph was analysed with SAFA Quant ${ }^{54}$.

SPR of CsiR/DNA interaction. Part of the csiD 5'-UTR containing the promoter region was amplified by PCR with primers MS178 and biotin-tagged MS177. The DNA was immobilized in TES buffer $(10 \mathrm{mM}$ Tris, $500 \mathrm{mM} \mathrm{NaCl}, 1 \mathrm{mM}$ EDTA $\mathrm{pH}=7.6)$ to reach $300 \mathrm{RU}$. Purified CsiR was diluted in running buffer $(50 \mathrm{mM}$ MOPS, $100 \mathrm{mM} \mathrm{NaCl}, 20 \mathrm{mM} \mathrm{KCl}, 2.5 \mathrm{mM} \mathrm{MgCl}, 0.1 \mathrm{mM} \mathrm{DTT}, 5 \mu \mathrm{g} / \mathrm{mL}$ sheared Salmon sperm DNA, $0.1 \mathrm{mg} / \mathrm{mL}$ BSA and $0.005 \%$ Tween $20 \mathrm{pH}=7.2$ ). Binding for indicated CsiR concentration was examined at a flow rate of $30 \mu \mathrm{L} / \mathrm{min}$. The surface was regenerated with a short pulse of $0.05 \%$ SDS. SPR was performed on a Biacore T200. The resulting sensograms were analyzed using the Biacore
Evaluation Software. Binding of $0.9 \mu \mathrm{M}$ CsiR was fitted kinetically assuming a 1:1 interaction.

\section{Data availability}

Data supporting the findings of this manuscript are available from the corresponding author upon reasonable request. A reporting summary for this Article is available as a Supplementary Information file. The presented crystal structures are available as PDBs 6GPE, 6HL8, 6GPN, and 6HL9.

Received: 27 June 2018 Accepted: 9 November 2018

Published online: 29 November 2018

\section{References}

1. Sabo, D. L., Boeker, E. A., Byers, B., Waron, H. \& Fischer, E. H. Purification and physical properties of inducible Escherichia coli lysine decarboxylase. Biochemistry 13, 662-670 (1974).

2. Lemonnier, M. \& Lane, D. Expression of the second lysine decarboxylase gene of Escherichia coli. Microbiology 144(Pt 3), 751-760 (1998).

3. Fothergill, J. C. \& Guest, J. R. Catabolism of L-lysine by Pseudomonas aeruginosa. J. Gen. Microbiol. 99, 139-155 (1977).

4. Metzner, M., Germer, J. \& Hengge, R. Multiple stress signal integration in the regulation of the complex sigma S-dependent csiD-ygaF-gabDTP operon in Escherichia coli. Mol. Microbiol. 51, 799-811 (2004).

5. Marschall, C. et al. Molecular analysis of the regulation of csiD, a carbon starvation-inducible gene in Escherichia coli that is exclusively dependent on sigma s and requires activation by cAMP-CRP. J. Mol. Biol. 276, 339-353 (1998).

6. Germer, J., Becker, G., Metzner, M. \& Hengge-Aronis, R. Role of activator site position and a distal UP-element half-site for sigma factor selectivity at a CRP/ H-NS-activated sigma(s)-dependent promoter in Escherichia coli. Mol. Microbiol. 41, 705-716 (2001).

7. Chance, M. R. et al. Structural genomics: a pipeline for providing structures for the biologist. Protein Sci. 11, 723-738 (2002).

8. Kalliri, E., Mulrooney, S. B. \& Hausinger, R. P. Identification of Escherichia coli YgaF as an L-2-hydroxyglutarate oxidase. J. Bacteriol. 190, 3793-3798 (2008).

9. Ye, D., Guan, K. L. \& Xiong, Y. Metabolism, activity, and targeting of D- and L-2-Hydroxyglutarates. Trends Cancer 4, 151-165 (2018).

10. Martinez, S. \& Hausinger, R. P. Catalytic mechanisms of Fe(II)- and 2oxoglutarate-dependent oxygenases. J. Biol. Chem. 290, 20702-20711 (2015).

11. Islam, M. S., Leissing, T. M., Chowdhury, R., Hopkinson, R. J. \& Schofield, C. J. 2-Oxoglutarate-dependent oxygenases. Annu. Rev. Biochem. 87, 585-620 (2018).

12. Struys, E. A., Jansen, E. E., Verhoeven, N. M. \& Jakobs, C. Measurement of urinary D- and L-2-hydroxyglutarate enantiomers by stable-isotope-dilution liquid chromatography-tandem mass spectrometry after derivatization with diacetyl-L-tartaric anhydride. Clin. Chem. 50, 1391-1395 (2004).

13. $\mathrm{Xu}, \mathrm{W}$. et al. Oncometabolite 2-hydroxyglutarate is a competitive inhibitor of alpha-ketoglutarate-dependent dioxygenases. Cancer Cell 19, 17-30 (2011).

14. Chowdhury, R. et al. The oncometabolite 2-hydroxyglutarate inhibits histone lysine demethylases. EMBO Rep. 12, 463-469 (2011).

15. Lohkamp, B. \& Dobritzsch, D. A mixture of fortunes: the curious determination of the structure of Escherichia coli BL21 Gab protein. Acta Crystallogr. D 64, 407-415 (2008).

16. Rzem, R., Van Schaftingen, E. \& Veiga-da-Cunha, M. The gene mutated in l2-hydroxyglutaric aciduria encodes 1-2-hydroxyglutarate dehydrogenase. Biochimie 88, 113-116 (2006).

17. Hudig, M. et al. Plants possess a cyclic mitochondrial metabolic pathway similar to the mammalian metabolic repair mechanism involving malate dehydrogenase and 1-2-Hydroxyglutarate dehydrogenase. Plant Cell Physiol. 56, 1820-1830 (2015).

18. Sharma, P., Teixeira de Mattos, M. J., Hellingwerf, K. J. \& Bekker, M. On the function of the various quinone species in Escherichia coli. Febs. J. 279, 3364-3373 (2012).

19. Unden, G. \& Bongaerts, J. Alternative respiratory pathways of Escherichia coli energetics and transcriptional regulation in response to electron acceptors. Biochim. Biophys. Acta 1320, 217-234 (1997).

20. Bekker, M. et al. The ArcBA two-component system of Escherichia coli is regulated by the redox state of both the ubiquinone and the menaquinone pool. J. Bacteriol. 192, 746-754 (2010)

21. Schneider, B. L. et al. The Escherichia coli gabDTPC operon: specific gammaaminobutyrate catabolism and nonspecific induction. J. Bacteriol. 184, 6976-6986 (2002).

22. Espinosa-Urgel, M. \& Ramos, J. L. Expression of a Pseudomonas putida aminotransferase involved in lysine catabolism is induced in the rhizosphere. Appl. Environ. Microbiol. 67, 5219-5224 (2001). 
23. Rohles, C. M., Giesselmann, G., Kohlstedt, M., Wittmann, C. \& Becker, J. Systems metabolic engineering of Corynebacterium glutamicum for the production of the carbon-5 platform chemicals 5 -aminovalerate and glutarate. Microb. Cell. Fact. 15, 154 (2016).

24. Shin, J. H. et al. Metabolic engineering of Corynebacterium glutamicum for enhanced production of 5-aminovaleric acid. Microb. Cell. Fact. 15, 174 (2016).

25. Schneider, B. L. \& Reitzer, L. Pathway and enzyme redundancy in putrescine catabolism in Escherichia coli. J. Bacteriol. 194, 4080-4088 (2012).

26. Samsonova, N. N., Smirnov, S. V., Altman, I. B. \& Ptitsyn, L. R. Molecular cloning and characterization of Escherichia coli K12 ygjG gene. BMC Microbiol. 3, 2 (2003).

27. Samsonova, N. N., Smirnov, S. V., Novikova, A. E. \& Ptitsyn, L. R. Identification of Escherichia coli K12 YdcW protein as a gammaaminobutyraldehyde dehydrogenase. FEBS Lett. 579, 4107-4112 (2005).

28. Jorge, J. M. P., Perez-Garcia, F. \& Wendisch, V. F. A new metabolic route for the fermentative production of 5 -aminovalerate from glucose and alternative carbon sources. Bioresour. Technol. 245, 1701-1709 (2017).

29. Kurihara, S. et al. A novel putrescine utilization pathway involves gammaglutamylated intermediates of Escherichia coli K-12. J. Biol. Chem. 280, 4602-4608 (2005).

30. Peekhaus, N. \& Conway, T. Positive and negative transcriptional regulation of the Escherichia coli gluconate regulon gene gnt $\mathrm{T}$ by GntR and the cyclic AMP (cAMP)-cAMP receptor protein complex. J. Bacteriol. 180, 1777-1785 (1998).

31. Jain, S. S. \& Tullius, T. D. Footprinting protein-DNA complexes using the hydroxyl radical. Nat. Protoc. 3, 1092-1100 (2008).

32. Revelles, O., Espinosa-Urgel, M., Fuhrer, T., Sauer, U. \& Ramos, J. L. Multiple and interconnected pathways for L-lysine catabolism in Pseudomonas putida KT2440. J. Bacteriol. 187, 7500-7510 (2005).

33. Zhang, M. et al. Increased glutarate production by blocking the glutaryl-CoA dehydrogenation pathway and a catabolic pathway involving 1-2hydroxyglutarate. Nat. Commun. 9, 2114 (2018).

34. Adkins, J., Jordan, J. \& Nielsen, D. R. Engineering Escherichia coli for renewable production of the 5-carbon polyamide building-blocks 5 aminovalerate and glutarate. Biotechnol. Bioeng. 110, 1726-1734 (2013).

35. Park, S. J. et al. Metabolic engineering of Escherichia coli for the production of 5-aminovalerate and glutarate as C5 platform chemicals. Metab. Eng. 16, 42-47 (2013)

36. Kranendijk, M., Struys, E. A., Salomons, G. S., Van der Knaap, M. S. \& Jakobs, C. Progress in understanding 2-hydroxyglutaric acidurias. J. Inherit. Metab. Dis. 35, 571-587 (2012).

37. Intlekofer, A. M. et al. L-2-Hydroxyglutarate production arises from noncanonical enzyme function at acidic pH. Nat. Chem. Biol. 13, 494-500 (2017).

38. Aghili, M., Zahedi, F. \& Rafiee, E. Hydroxyglutaric aciduria and malignant brain tumor: a case report and literature review. J. Neurooncol. 91, 233-236 (2009).

39. Rzem, R. et al. A gene encoding a putative FAD-dependent L-2hydroxyglutarate dehydrogenase is mutated in L-2-hydroxyglutaric aciduria. Proc. Natl Acad. Sci. USA 101, 16849-16854 (2004).

40. Hedlund, G. L., Longo, N. \& Pasquali, M. Glutaric acidemia type 1. Am. J. Med. Genet. C 142C, 86-94 (2006).

41. Sherman, E. A. et al. Genetic mapping of glutaric aciduria, type 3, to chromosome 7 and identification of mutations in c7orf10. Am. J. Hum. Genet. 83, 604-609 (2008)

42. Biagosch, C. et al. Elevated glutaric acid levels in Dhtkd1-/Gcdh- double knockout mice challenge our current understanding of lysine metabolism. Biochim. Biophys. Acta 1863, 2220-2228 (2017).

43. Wendel, U., Bakkeren, J., de Jong, J. \& Bongaerts, G. Glutaric aciduria mediated by gut bacteria. J. Inherit. Metab. Dis. 18, 358-359 (1995).

44. Baba, T. et al. Construction of Escherichia coli K-12 in-frame, single-gene knockout mutants: the Keio collection. Mol. Syst. Biol. 2, 20060008 (2006).

45. Kabsch, W. X. D. S. Acta Crystallogr. Sect. D 66, 125-132 (2010).

46. McCoy, A. J. et al. Phaser crystallographic software. J. Appl. Crystallogr. 40, 658-674 (2007).

47. Afonine, P. V. et al. Towards automated crystallographic structure refinement with phenix.refine. Acta Crystallogr. D 68, 352-367 (2012).

48. Emsley, P., Lohkamp, B., Scott, W. G. \& Cowtan, K. Features and development of Coot. Acta Crystallogr. D 66, 486-501 (2010).
49. Moriarty, N. W., Grosse-Kunstleve, R. W. \& Adams, P. D. Electronic ligand builder and optimization workbench (eLBOW): a tool for ligand coordinate and restraint generation. Acta Crystallogr. Sect. D 65, 1074-1080 (2009).

50. Carlone, G. M. \& Anet, F. A. Detection of menaquinone-6 and a novel methylsubstituted menaquinone- 6 in Campylobacter jejuni and Campylobacter fetus subsp. fetus. J. Gen. Microbiol. 129, 3385-3393 (1983).

51. Poderoso, J. J. et al. The reaction of nitric oxide with ubiquinol: kinetic properties and biological significance. Free Radic. Biol. Med. 26, 925-935 (1999).

52. Baum, R. H. \& Dolin, M. I. Isolation of 2-solanesyl-1,4-naphthoquinone from Streptococcus faecalis, 10 CL. J. Biol. Chem. 240, 3425-3433 (1965).

53. Bennett, B. D., Yuan, J., Kimball, E. H. \& Rabinowitz, J. D. Absolute quantitation of intracellular metabolite concentrations by an isotope ratiobased approach. Nat. Protoc. 3, 1299-1311 (2008).

54. Das, R., Laederach, A., Pearlman, S. M., Herschlag, D. \& Altman, R. B. SAFA: semi-automated footprinting analysis software for high-throughput quantification of nucleic acid footprinting experiments. RNA 11, 344-354 (2005).

55. Brechtel, C. E., Hu, L. \& King, S. C. Substrate specificity of the Escherichia coli 4 -aminobutyrate carrier encoded by gabP. Uptake and counterflow of structurally diverse molecules. J. Biol. Chem. 271, 783-788 (1996).

\section{Acknowledgements}

We thank Astrid Joachimi, Anna Heiler, Yuanhao Li, Dennis Kläge, Vera Hedwig, Malin Bein, Christoph Globisch, and the members of the Proteomics and NMR facilities of University of Konstanz for technical assistance and helpful discussions. This work was supported by an ERC Consolidator grant to J.S.H. We thank the Swiss Light Source synchrotron (Villigen, $\mathrm{CH}$ ) for access.

\section{Author contributions}

S.K., M.S., and J.S.H. conceived and designed this study. S.K., M.S., D.G., C.W., and D.S analysed the CsiD reactions. R.M.W. and O.M. crystallized and solved the CsiD structures. S.K. and N.M. characterized the LhgO reaction. S.K., M.S., and D.G. analysed the GabT/D, Sad/PuuE, and PatA/D reactions and carried out the isotope tracing experiment. M.S. analysed the ligand-dependency of CsiR. S.K., M.S., and J.S.H. wrote, and all authors commented on the manuscript.

\section{Additional information}

Supplementary Information accompanies this paper at https://doi.org/10.1038/s41467 018-07563-6.

Competing interests: The authors declare no competing interests.

Reprints and permission information is available online at http://npg.nature.com/ reprintsandpermissions/

Publisher's note: Springer Nature remains neutral with regard to jurisdictional claims in published maps and institutional affiliations.

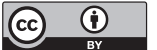

Open Access This article is licensed under a Creative Commons Attribution 4.0 International License, which permits use, sharing, adaptation, distribution and reproduction in any medium or format, as long as you give appropriate credit to the original author(s) and the source, provide a link to the Creative Commons license, and indicate if changes were made. The images or other third party material in this article are included in the article's Creative Commons license, unless indicated otherwise in a credit line to the material. If material is not included in the article's Creative Commons license and your intended use is not permitted by statutory regulation or exceeds the permitted use, you will need to obtain permission directly from the copyright holder. To view a copy of this license, visit http://creativecommons.org/ licenses/by/4.0/.

(C) The Author(s) 2018 